\title{
Localization Methods for a Mobile Robot in Urban Environments
}

\author{
Atanas Georgiev, Member, IEEE, and Peter K. Allen, Member, IEEE
}

\begin{abstract}
This paper addresses the problems of building a functional mobile robot for urban site navigation and modeling with focus on keeping track of the robot location. We have developed a localization system that employs two methods. The first method uses odometry, a compass and tilt sensor, and a global positioning sensor. An extended Kalman filter integrates the sensor data and keeps track of the uncertainty associated with it. The second method is based on camera pose estimation. It is used when the uncertainty from the first method becomes very large. The pose estimation is done by matching linear features in the image with a simple and compact environmental model. We have demonstrated the functionality of the robot and the localization methods with real-world experiments.
\end{abstract}

Index Terms-Localization, machine vision, mobile robots.

\section{INTRODUCTION}

$\mathbf{T}$ HE problem of building a functional autonomous mobile robot that can successfully and reliably interact with the real world is very difficult. It involves a number of issues such as proper design, choice of sensors, methods for localization, navigation, planning, and others, each of which is a challenge. A key factor of this complexity is the targeted environment of operation. The current state of mobile robotics is that most of the research has been focused on solving these issues indoors because of the slightly more predictable nature (e.g., flat horizontal floors, well-structured space partitioning, and smaller scale). On the other hand, many of the interesting applications are outdoors where fewer assumptions can be taken for granted.

In this paper, we target outdoor urban environments. These environments pose their own unique set of challenges that differentiate them from both the indoor and the open-space outdoor landscapes. On the one hand, they are usually too large to consider applying certain techniques that have achieved success indoors. On the other hand, typical outdoor sensors, such as global positioning system (GPS), have problems with reception around buildings.

While we have tried to keep the methods presented here general, we have focused on the development of our mobile robot system (Fig. 1) with a specific application in mind. The

Manuscript received May 2, 2003; revised January 26, 2004. This paper was recommended for publication by Associate Editor L. Parker and Editor S. Hutchinson upon evaluation of the reviewers' comments. This work was supported in part by the Office of Naval Research/Defense Advanced Research Projects Agency under MURI award ONR N00014-95-1-0601, and in part by the National Science Foundation under Grant EIA-97-29844 and Grant ANI-0099184.

The authors are with the Department of Computer Science, Columbia University, New York, NY 10027 USA (e-mail: atanas@cs.columbia.edu).

Digital Object Identifier 10.1109/TRO.2004.829506

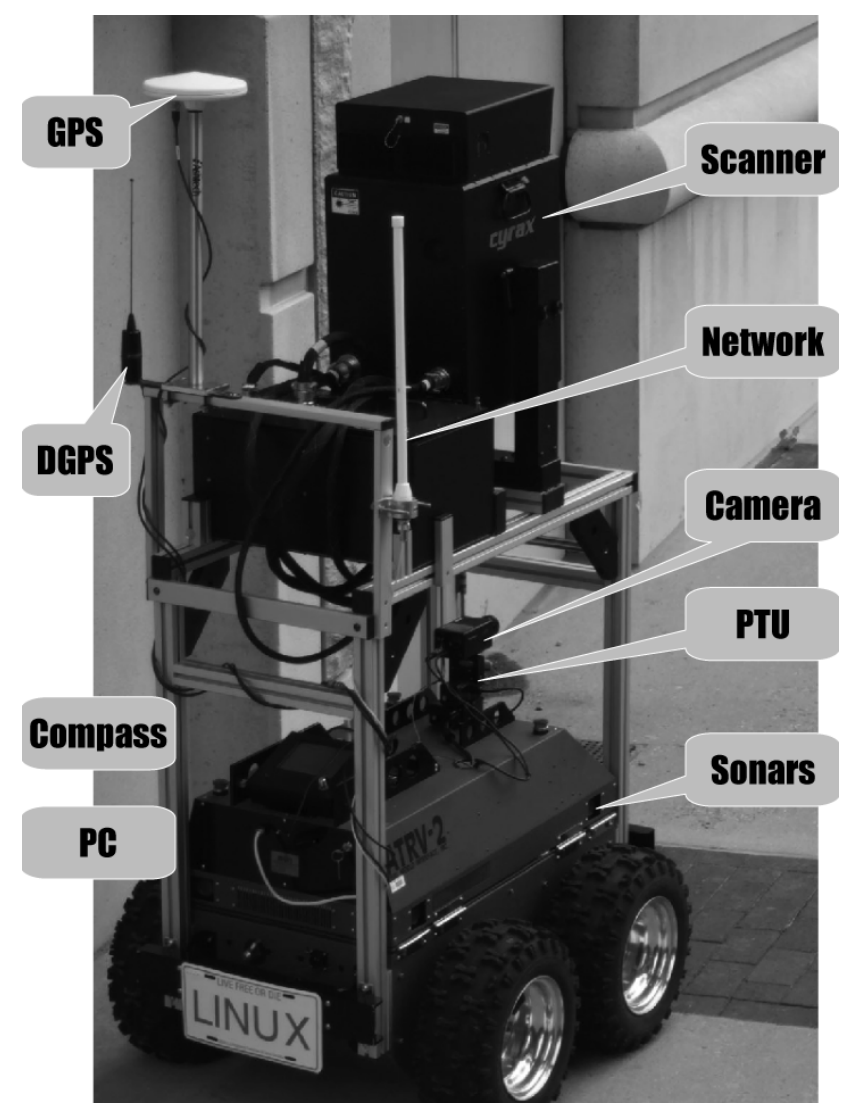

Fig. 1. Mobile platform used in this work.

AVENUE project at Columbia University's Robotics Laboratory (New York, NY) targets the automation of the urban site modeling process [1]. The main goal is to build geometrically accurate and photometrically correct models of complex outdoor urban environments. These environments are typified by large three-dimensional (3-D) structures that encompass a wide range of geometric shapes and a very large scope of photometric properties.

High-quality site models are needed in a variety of applications, such as city planning, urban design, fire and police planning, historical preservation and archaeology, virtual and augmented reality, geographic information systems, and many others. However, they are typically created by hand which is extremely slow and error-prone. The models built are often incomplete and updating them can be a serious problem. AVENUE addresses these issues by building a mobile system that will autonomously navigate around a site and create a model with minimum, if any, human interaction. 
The design and implementation of our mobile platform involved efforts that are related and draw from a large amount of existing work. For localization, dead reckoning has always been attractive because of its pervasiveness [2]-[4]. With the rapid development of technology, GPS receivers are quickly becoming the sensor of choice for outdoor localization [5]-[7]. Imaging sensors, such as charge-coupled device (CCD) cameras and laser range finders, have also become very popular mobile robot components [8]-[11]. Various methods for sensor integration and uncertainty handling have been proposed [12]-[16]. A very popular and successful idea is to exploit the duality between localization and modeling and address both issues in the same process, known as simultaneous localization and map building (SLAM) [14], [15], [17], [18]. Sensors and methods for indoor localization have been comprehensively reviewed in two books [19], [20]. Another excellent book presents case studies of successful mobile robot systems [21].

Researchers from the Australian Centre for Field Robotics have made significant progress toward using SLAM in outdoor settings. Dissanayake et al. have proved that a solution to the SLAM problem is possible and presented one such implementation [22]. Guivant et al. have further looked into optimizing the computational aspects of their algorithm and have applied it to an unstructured natural environment [23].

The problem of mobile robot localization in urban environments has been addressed by Talluri and Aggarwal by using feature correspondences between images taken by a camera on the robot and a CAD or similar model of its environment [24]. Chen and Shibasaki have improved on the accuracy and stability of GPS in urban areas by adding a camera and a gyro [25]. They have also relied on an environmental model obtained from a geodetic information system. Nayak has used a sensor suite consisting of four GPS antennas and a low-cost inertial measurement unit for localization of a car in urban areas; however, their resulting localization errors were on the order of meters, which is not acceptable for mobile robot navigation [26].

Our approach delivers a mobile robot system capable of operating autonomously under the challenges of urban environments. Whenever needed, we are making use of unique urban characteristics to facilitate the estimation of the robot location. Of all outdoor environments, urban areas seem to possess the most structure in the form of buildings. The laws of physics dictate common architectural design principles according to which the horizontal and vertical directions play an essential role, and parallel line features are abundant. The system presented here takes advantage of these characteristics. We believe that the main contributions of our study are the practical realization of a functioning mobile robot for site navigation and modeling and a novel method of supplementing odometry and GPS with visual image processing to allow accurate localization of the robot under varying conditions, including odometry error and GPS degradation.

The rest of this paper is organized as follows. The next section briefly describes our mobile system and software architecture. Section III describes the first of our localization methods, based on odometry, a digital compass module, and global positioning. Section IV presents our vision-based localization methods. Experimental results are shown in Section V, and in Section VI we conclude with a summary and a discussion on future extensions of this work.

\section{SySTEM DESIGN AND IMPLEMENTATION}

The mobile robot used as a test bed for this work is an ATRV-2 model manufactured by iRobot (Fig. 1). It has built-in odometry and 12 sonars and carries a regular PC on-board. For modeling, we have installed a Cyrax 2500 laser range scanner with a range of up to $100 \mathrm{~m}$. For navigation, we have added a Honeywell HMR3000 digital compass module with an integrated roll-pitch sensor, an Ashtech GG24C GPS+GLONASS ${ }^{1}$ receiver which is accurate down to $1 \mathrm{~cm}$ in real-time kinematic (RTK) mode, and a color CCD camera mounted on a pan-tilt unit (PTU). Communication with the robot is done via a $802.11 \mathrm{~b}$ wireless network.

The combination of dead reckoning and GPS is known to be beneficial. GPS tends to exhibit an unstable high-frequency behavior manifested by sudden "jumps" of the position estimates but is fairly reliable over a longer period of time. On the other hand, dead-reckoning sensors drift gradually and rarely suffer the sudden jump problem.

The camera is needed to address some of the limitations of GPS operation that are quite consistent in urban areas. Tall buildings in the vicinity may obstruct the clear view to the satellites, the SNR could be attenuated by trees or large structures standing in the way or one may encounter signal reflections or multipath. The result is unstable, wrong, or has no position fixes in some areas. However, due to the nature of urban sites and the overall goal of AVENUE, it is mostly around buildings that degradation in GPS performance is likely to occur. With the addition of a camera, we make use of this by exploiting typical urban characteristics, such as abundance of linear features, parallel lines, and horizontal and vertical principal directions, which are relatively easy to find and process using computer vision techniques.

Our system architecture (Fig. 2) addresses the various tasks associated with an autonomous navigation and modeling in a modular and distributed fashion. Its main building blocks are concurrently executing distributed software components which can communicate across the network. The robot is designed to operate according to the following scenario. Its task is to go to desired locations and acquire requested 3-D scans and images of selected buildings. The locations are determined by the sensor planning system and are used by the path planning system to generate reliable trajectories which the robot follows. When the rover arrives at the target location, it uses the sensors to acquire the scans and images and forward them to the modeling system. The modeling system registers and incorporates the new data into the existing partial model of the site (which in the beginning could be empty). After that, the view planning system decides upon the next best data acquisition location and the above steps repeat. The process starts from a certain location and gradually expands the area it has covered until a complete model of the site is obtained. The user interface (Fig. 3) provides a comprehensive view of the robot location and activities within its

${ }^{1}$ Throughout this paper, we will use GPS to designate either or both of the U.S. NAVSTAR GPS and the Russian GLONASS infrastructures. 


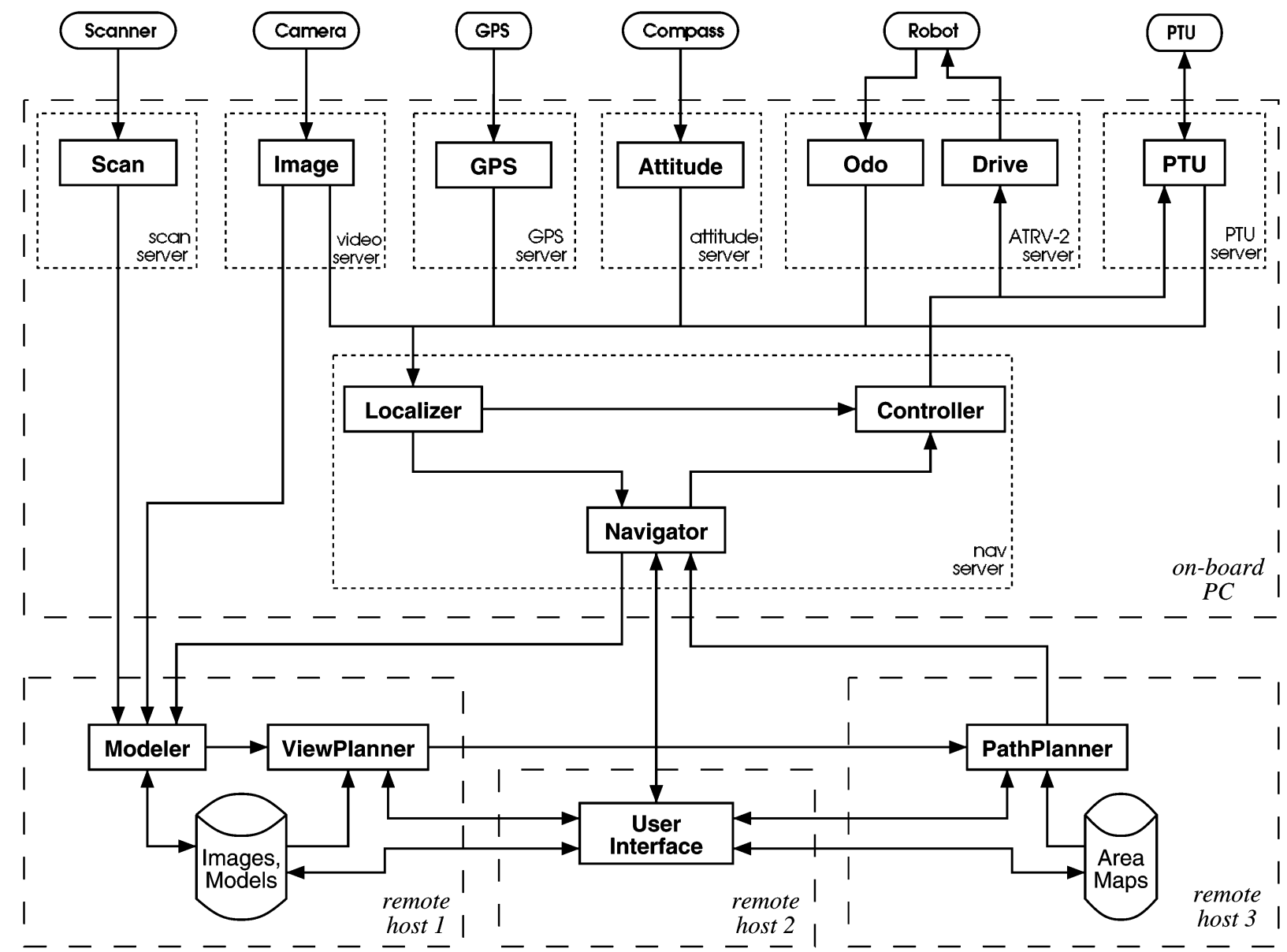

Fig. 2. System architecture. Solid rectangles represent components, dotted rectangles are processes, and dashed rectangles group processes running on the same machine. The arrows show the data flow between components.

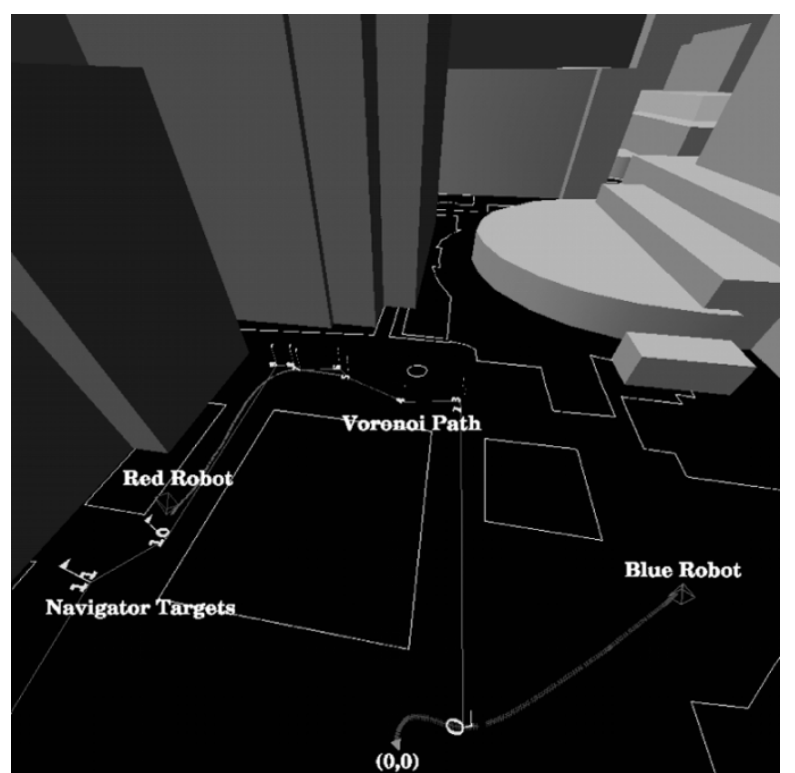

Fig. 3. User interface. The window shows the outlines of the 2-D map and simplified 3-D models of buildings. The actual trajectories of two robots are visible along with the planned path for one of them (denoted with flags).

environment and allows the user to monitor the progress and exercise control of the mission.
The entire task is quite complex and requires the solution of a number of additional fundamental problems which we have addressed in our project. Due to limited space, we refer the reader to [27]-[29].

\section{LOCALIZATION IN OPEN SPACE}

The first of our localization methods is designed for real-time usage in open-space outdoor environments. It uses the built-in robot odometry and the added digital compass/tilt sensor and GPS receiver. We exploit the redundancy in the measurements of these sensors to fuse their estimates using an extended Kalman filter shown in Fig. 4 [30].

The control input to the robot consists of the scalar translational velocity $v(t)$ and the scalar angular velocity $\omega(t)$. Due to the kinematics of the robot (Fig. 5), the translational velocity vector $\mathbf{v}(\mathbf{t})$ always points forward and the angular velocity vector $\mathbf{w}(\mathbf{t})$ always points up. With respect to the robot coordinate frame, these velocities can be expressed as $\mathbf{v}^{\prime}(\mathbf{t})=$ $v(t) \mathbf{e}_{\mathbf{x}}$ and $\mathbf{w}^{\prime}(\mathbf{t})=\omega(t) \mathbf{e}_{\mathbf{z}}$, where $\mathbf{e}_{\mathbf{x}}=[1,0,0]^{T}$ and $\mathbf{e}_{\mathbf{z}}=$ $[0,0,1]^{T}$.

Denote the position of the robot at time $t$ by $\mathbf{p}(\mathbf{t})=$ $[x(t), y(t), z(t)]^{T}$ and its orientation as expressed by the Euler angles by $\mathbf{r}(t)=[\varphi(t), \theta(t), \psi(t)]^{T}$. Let $R(\mathbf{r})=\operatorname{Rot}_{z}(\psi) \operatorname{Rot}_{y}(\theta) \operatorname{Rot}_{x}(\varphi)$ be the roll-pitch-yaw matrix corresponding to the Euler angles $\mathbf{r}$, let $R^{-1}(A)$ be 




Fig. 4. Diagram of the extended Kalman filter configuration.

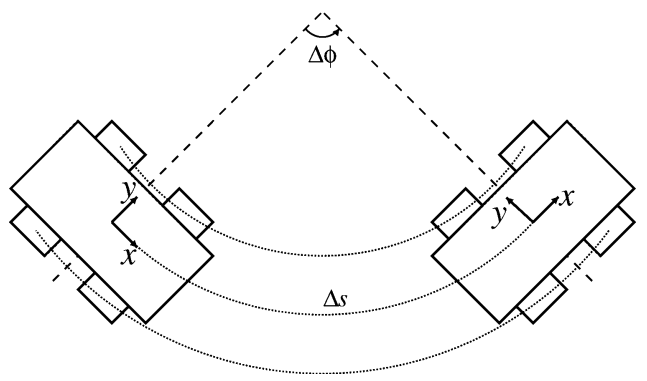

Fig. 5. ATRV kinematics.

the function that returns the Euler angles of a roll-pitch-yaw matrix $A$, and let $M(A)$ be the standard matrix that transforms 3 -D angular rate to Euler angle time derivatives. Then, in the world coordinate frame, the velocities of the robot are $\mathbf{v}(\mathbf{t})=v(t) R(\mathbf{r}(\mathbf{t})) \mathbf{e}_{\mathbf{x}}$ and $\mathbf{w}(\mathbf{t})=\omega(t) R(\mathbf{r}(\mathbf{t})) \mathbf{e}_{\mathbf{z}}$ and our system model for the robot motion becomes (with implicit dependence on $t$ )

$$
\dot{\mathbf{x}}=\left[\begin{array}{c}
\dot{\mathbf{p}} \\
\dot{\mathbf{r}}
\end{array}\right]=\left[\begin{array}{c}
v R(\mathbf{r}) \mathbf{e}_{x} \\
\omega M(\mathbf{r}) \mathbf{e}_{z}
\end{array}\right]+\mathbf{u}_{\mathbf{m}}
$$

where state vector $\mathbf{x}=[x, y, z, \varphi, \theta, \psi]^{T}$ is the robot pose and the vector $\mathbf{u}_{\mathbf{m}}$ is added noise accounting for system misrepresentation.

Let $\tilde{\mathbf{x}}$ be an estimate of the robot pose and let the error of this estimate be $\delta \mathbf{x}=\mathbf{x}-\tilde{\mathbf{x}}$. Applying first-order Taylor series approximation, we linearize equation (1) about $\tilde{\mathbf{x}}$ to yield

$$
\delta \dot{\mathbf{x}}=\mathbf{F} \delta \mathbf{x}+\mathbf{u}
$$

where $\mathbf{F}$ is a $6 \times 6$ matrix obtained from the partial derivatives in (1) and $\mathbf{u}$ includes both the system misrepresentation term from (1) and the uncertainty in the estimate $\tilde{\mathbf{x}}$. We assume it to be zero-mean Gaussian white noise, $\mathbf{u} \sim N(0, \mathbf{Q})$. Next, we solve the differential equation and discretize in time to obtain the state transition matrix

$$
\mathbf{\Phi}_{k} \approx \mathbf{I}+\left(t_{k+1}-t_{k}\right) \mathbf{F}
$$

and the Kalman filter prediction equations

$$
\begin{aligned}
\delta \hat{\mathbf{x}}_{\mathbf{k}+\mathbf{1}}^{-} & =\boldsymbol{\Phi}_{\mathbf{k}} \delta \hat{\mathbf{x}}_{k}^{+} \\
\hat{\mathbf{P}}_{\mathbf{k}+\mathbf{1}}^{-} & =\boldsymbol{\Phi}_{\mathbf{k}} \hat{\mathbf{P}}_{\mathbf{k}}^{+} \boldsymbol{\Phi}_{\mathbf{k}}^{\mathbf{T}}+\mathbf{Q}_{\mathbf{k}} .
\end{aligned}
$$

The notation adopted here is that the hat denotes values estimated by the filter, the minus superscript denotes predicted values, the plus superscript denotes corrected values, and the subscript denotes the time interval. The matrix $\hat{\mathbf{P}}_{\mathbf{k}}$ is the covariance of the estimated error state $\delta \hat{\mathbf{x}}_{\mathbf{k}}$ and $\mathbf{Q}_{\mathbf{k}}$ is the noise covariance for the time period $\left[t_{k}, t_{k+1}\right]$. Details of the derivations can be found in [31].

We obtain the reference trajectory $\tilde{\mathbf{x}}$ from odometry. Counts from encoders on the axles are regularly sampled by the robot firmware and converted into angular displacement $\Delta \phi_{i}$ and travel distance $\Delta s_{i}$ during the sampling interval. Note that the odometry sampling times do not necessarily coincide with the update times of the Kalman filter (which happen when measurements from the other sensors become available). Since we only have discrete samples, we need to interpolate to obtain a continuous trajectory. For a sampling interval $\left[t_{i}^{\prime}, t_{i+1}^{\prime}\right]$ of the odometry, we have

$$
\begin{aligned}
\Delta \phi_{i}^{\prime}(t) & =\Delta \phi_{i}\left(t-t_{i}^{\prime}\right) /\left(t_{i+1}^{\prime}-t_{i}^{\prime}\right) \\
{\left[\begin{array}{c}
\Delta x_{i}^{\prime}(t) \\
\Delta y_{i}^{\prime}(t)
\end{array}\right] } & =\Delta s_{i} \frac{t-t_{i}^{\prime}}{t_{i+1}^{\prime}-t_{i}^{\prime}} \int_{t_{i}^{\prime}}^{t}\left[\begin{array}{l}
\cos \left(\Delta \phi_{i}^{\prime}(t)\right) \\
\sin \left(\Delta \phi_{i}^{\prime}(t)\right)
\end{array}\right] d \tau
\end{aligned}
$$

where the displacements $\Delta x_{i}^{\prime}(t), \Delta y_{i}^{\prime}(t)$, and $\Delta \phi_{i}^{\prime}(t)$ are with respect to the robot pose $\tilde{\mathbf{x}}_{\mathbf{i}}^{\prime}=\left[\tilde{\mathbf{p}}_{\mathbf{i}}^{\prime}, \tilde{\mathbf{r}}_{\mathbf{i}}^{\prime}\right]^{T}$ at time $t_{i}^{\prime}$ and necessarily assume a planar local motion. In cases where we can assume that the ground is flat, the reference plane for each sampling period will be the same (the ground) and a running total of these displacements will give us the overall odometry estimate of the robot pose in 2-D. Here, we are interested in the full 3-D pose, however, and need to account for the robot pitch and roll as follows:

$$
\begin{aligned}
\tilde{\mathbf{p}}(\mathbf{t}) & =\tilde{\mathbf{p}}_{\mathbf{i}}^{\prime}+R\left(\tilde{\mathbf{r}}_{\mathbf{i}}^{\prime}\right)\left[\Delta x_{i}^{\prime}(t), \Delta y_{i}^{\prime}(t), 0\right]^{T} \\
\tilde{\mathbf{r}}(\mathbf{t}) & =R^{-1}\left(R\left(\tilde{\mathbf{r}}_{\mathbf{i}}^{\prime}\right) \operatorname{Rot}_{z}\left(\Delta \phi_{i}^{\prime}(t)\right)\right) .
\end{aligned}
$$

If a new measurement comes from another sensor at time $t_{k} \in$ $\left[t_{i}^{\prime}, t_{i+1}^{\prime}\right]$, then that measurement is fed through the filter together with the odometry pose $\tilde{\mathbf{x}}_{\mathbf{k}}^{-}$as computed from (8) and (9) and the resulting error estimate $\delta \hat{\mathbf{x}}_{\mathbf{k}}^{+}$is transferred to $\tilde{\mathbf{x}}_{\mathbf{k}}^{-}$to produce a corrected odometry estimate $\tilde{\mathbf{x}}_{\mathbf{k}}^{+}$. Then, the remaining portion of the displacements $\Delta \phi_{i}$ and $\Delta s_{i}$ (which occurred during $\left.\left[t_{k}, t_{i+1}^{\prime}\right]\right)$ is added to the odometry according to (8) and (9), only this time using the corrected odometry estimate $\tilde{\mathbf{x}}_{\mathbf{k}}^{+}$as a reference. In essence, whenever the filter updates the odometry trajectory, it updates the local reference plane of motion used in the subsequent odometry iterations.

We would like to point out that regardless of whether a 2-D or a 3-D odometry formulation is used, minute measurement errors during each sampling interval accumulate and reach a point when they can no longer be neglected. Typically, a robot may 
be able to accurately traverse a few meters, but after that relying on odometry becomes impractical. These errors cannot be avoided without some means of more direct measurements, such as GPS. It is nevertheless possible to address them. Systematic errors, which are ones caused by kinematic imperfections of the vehicle, can be estimated and compensated for using accurate calibration. We have used the UMBmark method to do so [2]. Generally, the point of performing such a calibration is to make sure the systematic errors will be reduced to negligible compared to the nonsystematic ones. Nonsystematic errors (e.g., due to slippery spots or over-acceleration) are by definition random and nonpredictable. They are accounted for by the system disturbance vector $\mathbf{u}$ in (2). In essence, we have modeled their "average" behavior so that we keep track of the uncertainty in the robot pose estimates. Our model is a Gaussian probability distribution with standard deviation proportional to the distance traveled. While technically not exact, this is a good enough approximation over a short distance until the robot obtains external observations of its location.

Although (8) and (9) work in 3-D, they cannot produce an accurate $3-\mathrm{D}$ pose based solely on odometry, because odometry lacks the necessary observational power. To provide a full three degrees of freedom (DOF) in orientation, we have added a compass and tilt sensor module which reports the heading (yaw), pitch, and roll angles. It is mounted level on the robot and is calibrated for magnetic variation and deviation. The observation model is quite simple as it is already linear, given as follows:

$$
\begin{aligned}
\mathbf{z}_{\mathbf{k}}^{\mathbf{c}} & =\left[\varphi_{k}^{c}, \theta_{k}^{c}, \psi_{k}^{c}\right]^{T} \\
\mathbf{H}_{\mathbf{k}}^{\mathbf{c}} & =\left[\begin{array}{cccccc}
0 & 0 & 0 & 1 & 0 & 0 \\
0 & 0 & 0 & 0 & -1 & 0 \\
0 & 0 & 0 & 0 & 0 & -1
\end{array}\right] \\
\mathbf{R}_{\mathbf{k}}^{\mathbf{c}} & =\operatorname{diag}\left(\sigma_{t}^{2}, \sigma_{t}^{2}, \sigma_{h}^{2}\right)
\end{aligned}
$$

where $\mathbf{z}_{\mathbf{k}}^{\mathbf{c}}$ is the sensor measurement vector, $\mathbf{H}_{\mathbf{k}}^{\mathbf{c}}$ is the observation matrix, and $\mathbf{R}_{\mathbf{k}}^{\mathbf{c}}$ is the observation uncertainty. The negative signs in $\mathbf{H}_{\mathbf{k}}^{\mathbf{c}}$ are due to the sensor coordinate system being oriented forward-right-down while the robot frame is forwardleft-up. We assume a Gaussian distribution of the measurement error with tilt and heading standard deviations, $\sigma_{t}$ and $\sigma_{h}$, based on the manufacturer's specifications. The sensor data is used to update the state vector according to the standard Kalman filter equations

$$
\begin{aligned}
\mathbf{K}_{\mathbf{k}+\mathbf{1}} & =\hat{\mathbf{P}}_{\mathbf{k}+\mathbf{1}}^{-} \mathbf{H}_{\mathbf{k}+\mathbf{1}}^{\mathbf{T}}\left(\mathbf{H}_{\mathbf{k}+\mathbf{1}} \hat{\mathbf{P}}_{\mathbf{k}+\mathbf{1}}^{-} \mathbf{H}_{\mathbf{k}+\mathbf{1}}^{\mathbf{T}}+\mathbf{R}_{\mathbf{k}+\mathbf{1}}\right)^{-1} \\
\delta \mathbf{z}_{\mathbf{k}+\mathbf{1}} & =\mathbf{z}_{\mathbf{k}+\mathbf{1}}-\mathbf{H}_{\mathbf{k}+\mathbf{1}} \tilde{\mathbf{x}}_{\mathbf{k}+\mathbf{1}}^{-} \\
\delta \hat{\mathbf{x}}_{\mathbf{k}+\mathbf{1}}^{+} & =\boldsymbol{\delta} \hat{\mathbf{x}}_{\mathbf{k}+\mathbf{1}}^{-}+\mathbf{K}_{\mathbf{k}+\mathbf{1}}\left[\boldsymbol{\delta} \mathbf{z}_{\mathbf{k}+\mathbf{1}}-\mathbf{H}_{\mathbf{k}+\mathbf{1}} \boldsymbol{\delta} \hat{\mathbf{x}}_{\mathbf{k}+\mathbf{1}}^{-}\right] \\
\hat{\mathbf{P}}_{\mathbf{k}+\mathbf{1}}^{+} & =\left(\mathbf{I}-\mathbf{K}_{\mathbf{k}+\mathbf{1}} \mathbf{H}_{\mathbf{k}+\mathbf{1}}\right) \hat{\mathbf{P}}_{\mathbf{k}+\mathbf{1}}^{-}
\end{aligned}
$$

The GPS receiver is very useful because it limits the error accumulated by the dead reckoning sensors. It provides periodic fixes of the location of the GPS antenna, $\mathbf{z}_{\mathbf{k}}^{\mathbf{g}}=\left[x_{k}^{g}, y_{k}^{g}, z_{k}^{g}\right]^{T}$. Since the antenna is placed at location $\Delta \mathrm{p}^{\mathrm{g}}$ with respect to the robot coordinate frame, the observation model is not linear, shown as follows:

$$
\mathbf{h}^{\mathbf{g}}(\mathbf{x})=\mathbf{p}+R(\mathbf{r}) \Delta \mathbf{p}^{\mathbf{g}}
$$

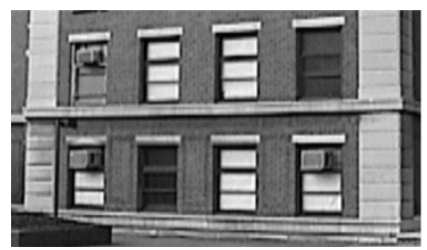

(a)

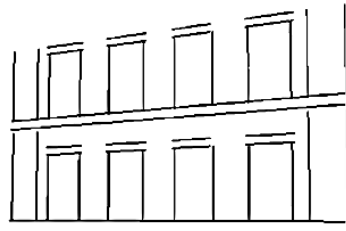

(b)
Fig. 6. (a) A building facade and (b) a sample model of it.

The fix is incorporated into the filter via (13)-(16) where the observation matrix is $\mathbf{H}_{\mathbf{k}}^{\mathbf{g}}=\left.\nabla \mathbf{h} \mathbf{g}(\mathbf{x})\right|_{\hat{\mathbf{x}}_{\mathrm{k}}^{-}}$and the measurement uncertainty $\mathbf{R}_{\mathbf{k}}^{\mathbf{g}}$ is the one reported by the receiver.

The GPS is the only sensor in this method that makes absolute position measurements and thus the overall accuracy of the method depends strongly on the accuracy of the GPS fixes. If GPS quality deteriorates, the uncertainty in the pose estimates may become too large. In such cases, positioning data are needed from additional sensors. However, in order to seek such data, there has to be a way to detect these situations. This is done by monitoring the variance-covariance matrix representing the uncertainty in the Kalman filter. Each of the diagonal elements of this matrix reflects the variance of the corresponding element (position or orientation coordinate) of the state vector. Whenever a new GPS update is processed by the filter, a test is performed to check if the variance associated with the robot position is greater than a threshold. If so, we consider this as an indication that additional data are needed and attempt to use the visual localization method described next. Only the uncertainly in position is considered, because if the orientation is wrong it will quickly cause the position error to also increase.

\section{VISUAL LOCALIZATION}

To expand the working range of our localization system, it is sufficient to provide occasional "on-demand" updates only when the open-space configuration fails. Visual pose-estimation algorithms are well poised to do that. By acting less frequently and on demand, they can be allowed more time for image processing operations which can be used to increase the robustness of the overall system.

This is the underlying idea in the use of our visual localization system. As the robot moves, it uses the open-space localization method described in the previous section to keep track of its pose along with the uncertainty. As long as it is confident in these pose estimates, no attempts are made to use vision. If the confidence becomes low, then the robot is allowed to stop and compute a more accurate estimate using the vision-based pose estimation method described in this section.

\section{A. Environmental Model}

The visual pose estimation is based on matching an image of a building taken by the camera with a model. The environmental model we use is a database of smaller scale facade models. Each facade model depicts the features of a near-planar region around a building facade (Fig. 6). The features modeled are dominant 


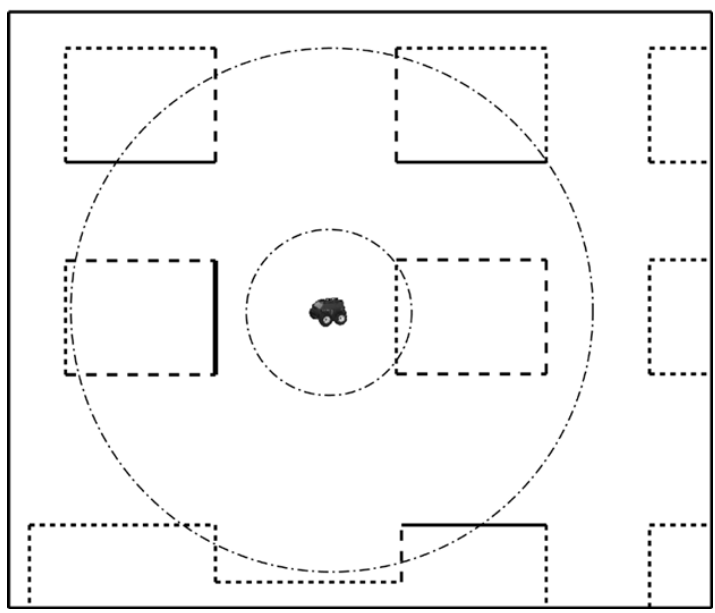

Fig. 7. Choosing a model: a top-down view of modeled facades of buildings are shown on the map. The two circles show the minimum and maximum distance allowed. The dotted lines are models that are outside of this range. The dashed lines are models that are within the range but are viewed at a very low (or negative) angle. The solid lines are good to use. The thick one is chosen because it is closest to the robot.

straight lines - typical and abundant in a human-made environments. All lines are finite segments represented by the 3-D coordinates of their endpoints in a local coordinate system, which is registered with the "world" coordinate system for the entire site.

In order to be useful, each facade model needs to capture enough features to provide sufficient information for the robot to find its pose. The number of features varies across buildings but, beyond a certain limit, adding more detail quickly reaches the point of diminishing return. There is no need to model every facade or every building either-what is needed is that enough building facades are modeled to allow continuous localization throughout the area of interest. Hence, the model we use is simple and compact. The model used in this paper was created by hand, however, our approach on how to create the models automatically is discussed in Section VI.

\section{B. Choosing a Model to Use}

When visual pose estimation is attempted, a rough estimate of the robot pose is available from the other sensors. This estimate is used to search the model database for the most appropriate building facade to use for visual localization. This is done in two steps according to two criteria: distance and viewing angle (Fig. 7).

The first step is to scan through the model database index to determine the facade models that are within a good distance from the robot. Both minimal and maximal limits are imposed: if a building is too close, it may not have enough visible features on the image; if it is too far, the accuracy of the result may be low because of the fixed camera resolution.

The second step is to eliminate facade models from the first step based on the viewing angle (ranging from $90^{\circ}$ for an anterior view to $-90^{\circ}$ for a posterior view). Only models that are viewable under a sufficiently large angle are considered. This eliminates both facades that are not visible (negative angles) and ones that are visible at too low an angle to produce a stable match with the image.
The models that successfully pass this two-step selection process form the set of good candidate models to use. Any subset of this set can be used in the pose estimation step. As the processing time is not trivial, however, we choose to use only the one that is closest to the robot. Because of the finite resolution of the camera, this choice is likely to provide the most accurate result.

Finally, the pan-tilt head holding the camera is turned toward the chosen facade and an image is taken. The pan and tilt angles are computed from the known rough pose of the robot so that the camera faces the center of mass of the model. In practice, the final orientation of the camera is different from the ideal one because of the uncertainty in the current pose. However, for the small distances involved and the typical accuracy of the pose estimates, the resulting orientation error of the camera is usually within the tolerance of the processing steps that follow. Further, since the camera is aimed at the center of the model, any small deviation will have minimal effect.

\section{Pose Estimation}

At this stage, a pair of an image and a model of the building facade are available and the task is to determine the pose of the robot. Since the camera is tracked by the pan-tilt unit rigidly affixed to the robot, if the camera pose is known, then the pose of the robot can be easily derived. Thus, the focus from now on is on the computation of the camera pose.

The pose computation is done by matching identical linear features in the image and the model. We have adopted a probabilistic approach following the well-known RANSAC paradigm first introduced by Fischler and Bolles [32]. The method consists of the following five steps. The first step is executed once, while the rest of the steps are repeated in a loop with a predetermined number of iterations.

1) Preparation: The purpose of the preparation step is to obtain the line segments and do some preprocessing necessary for the steps that follow. The image of the building is processed to obtain the 2-D line segments. A Canny edge detector is applied to locate edges, and then an incremental line-fitting technique is used to connect them in straight line segments.

To reduce the computational burden in the following steps, collinear lines are merged and ones shorter than a given threshold are discarded. Details about this process are presented in the Appendix.

2) Sampling and Pose Candidate Computation: The idea behind RANSAC is to solve the pose estimation problem a number of times using randomly chosen matches between a minimum number of 2-D and 3-D line segments. The minimum number of matching pairs in this case is three: the problem has six unknowns (three for position and three for orientation of the camera) and each matching pair of segments provides a 2-DOF constraint. The equations are nonlinear and more than one solution is possible, however, the initial pose estimate from the other sensors is usually sufficient to converge to the correct one. Thus, in the sampling step, we randomly select three pairs of lines and, based on this selection, compute an estimate for the camera pose. 


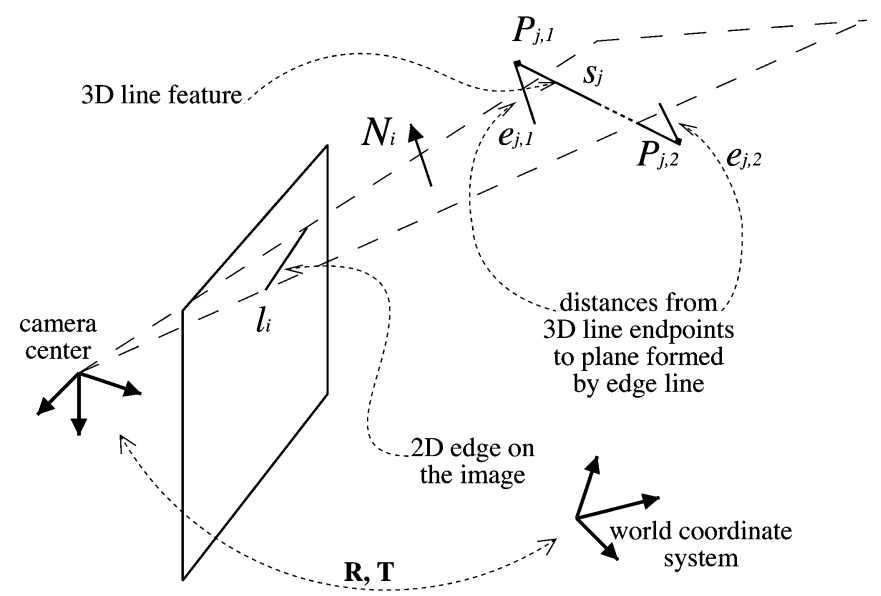

Fig. 8. Error metric used for pose estimation.

The camera pose candidate is found by using the pose estimation method proposed by Kumar and Hanson [33]. A perspective camera model is used and the calibration parameters of the camera are assumed to be known. An error function is composed and minimized that quantifies the misalignment of the 3-D line and its matching 2-D line from the sample. For each 2-D line $l_{i}$, consider the plane that is formed by that line and the camera center of projection (Fig. 8). Let the normal to that plane be $N_{i}$. Suppose $l_{i}$ is matched with the 3-D line segment $s_{j}$ whose endpoints $P_{j, 1}$ and $P_{j, 2}$ have world coordinates $\mathbf{p}_{\mathbf{j}, \mathbf{1}}$ and $\mathbf{p}_{\mathbf{j}, \mathbf{2}}$. If $R$ and $T$ are the rotation and translation that align the world coordinate system with that of the camera, then

$$
d_{i, j}=\left(N_{i} \cdot\left(R \cdot \mathbf{p}_{\mathbf{j}, \mathbf{1}}+T\right)\right)^{2}+\left(N_{i} \cdot\left(R \cdot \mathbf{p}_{\mathbf{j}, \mathbf{2}}+T\right)\right)^{2}
$$

is the sum of squared distances of the endpoints of $s_{j}$ to the plane formed by $l_{i}$ (Fig. 8). The error function that is minimized is the sum of $d_{i, j}$ for the three matching pairs

$$
E(R, T)=\sum_{i, j \in \text { Matches }} d_{i, j}
$$

This function is minimized with respect to the $6 \mathrm{DOF}$ for the camera pose: three for the rotation $R$ and three for the translation vector $T$. The computation follows the method proposed by Horn [34].

3) Pose Candidate Refinement: The pose candidate refinement step uses the consensus set to fine tune the estimate. The consensus set is the set of all matching pairs of 2-D edge segments from the image and 3-D line segments from the model that agree with the initially computed pose candidate.

For each 3-D line segment in the model, a neighborhood of its projection on the image is searched for 2-D edges and their distance from the 3-D line segment is computed according to (18). The 2-D edge with the smallest distance is taken to be the match, if that distance is less than a threshold and if the 2-D line is not closer to another 3-D line. If no such 2-D edge is found, then the 3-D line segment is assumed to have no match.

The consensus set is used together with (19) to compute a better pose estimate. This is done iteratively a number of times (between 1 and 4) starting with a large value for the consensus threshold and gradually decreasing it. The large initial value for the threshold makes sure that a roughly correct consensus set will be generated initially which will be later refined to eliminate the false positives and increase the accuracy. The result of the last iteration is the pose candidate that is evaluated in the next step.

4) Pose Candidate Evaluation: The quality of each pose candidate is judged by a metric $q(R, T)$ which quantifies the amount of support for the pose by the matches between the model and the edge. The idea is to check what portion of the model is covered by matching edge lines. The larger the coverage, the better the pose candidate. Ideally, the entire visible portion of the model should be covered by matching 2-D edge lines.

After the last refinement iteration, the consensus set contains pairs of matching 3-D lines from the model and 2-D lines from the edges. Consider one 3-D line $s_{j}$ in the consensus set and its matching 2-D counterpart $l_{i}$. Let the perspective projection of $s_{j}$ onto the image be $s_{j}^{\prime}$ and the orthogonal projection of $l_{i}$ onto $s_{j}^{\prime}$ be $l_{i}^{\prime}$. We set the contribution $c\left(s_{j}\right)$ of the match between $s_{j}$ and $l_{i}$ to the length of the overlap between $s_{j}^{\prime}$ and $l_{i}^{\prime}$. Thus, the total portion of the model covered by matching line edges in the image is

$$
C(R, T)=\sum_{s_{j} \in \text { Model }} c\left(s_{j}\right) .
$$

The dependence on $R$ and $T$ is implicit as the consensus set and the projections $s_{j}^{\prime}$ depend on the pose.

Note that the coverage is a quantity which is computed in 2-D space. As such, it depends on the scale of the model as well. If the camera moves away from the building, the visible size of the model will diminish and $C(R, T)$ will decrease even if the match is perfect. Hence, normalization needs to take place.

There are two ways to normalize the coverage: divide by the total projected length of the model or divide only by the visible projected length. The former approach will tend to underrate the correct pose in cases when the model is slightly outside of the field of view. The latter approach will do fine in such cases but will overrate poses for which very little of the model is visible and the visible portion can easily match arbitrary edge lines. We have chosen to use the latter method and compute the pose evaluation metric as

$$
q(R, T)=C(R, T) / V(R, T)
$$

where $V(R, T)$ is the total length of the visible projection of the model on the image.

To avoid the pitfalls of choosing an overrated pose, we use three criteria by which eliminate a given pose candidate from consideration.

1) If the pose candidate is outside of a validation gate, it is immediately rejected as unlikely. The validation gate is determined by the total state estimate of the extended Kalman filter.

2) If the visible portion of the model on the image is less than a threshold, the pose is also rejected as there is not sufficient basis to evaluate it, even if it is the correct one. If this is the case, the entire localization step is likely to fail, because the camera was pointed way off target. 
3) If the value $q(R, T)$ for the current pose candidate is less than a threshold, the pose is also rejected as there is insufficient support for it.

Of all the pose candidates that pass the three tests, the one with the highest score after the loop is the best one and is accepted to be the correct pose. It is used along with an empirically obtained covariance matrix for each model to update the Kalman filter estimate. If no good pose is found, the visual localization step fails. This is not fatal, however, as the robot simply moves a little further along its route and attempts another visual localization step. This is repeated until either the visual localization succeeds or the GPS picks up a good signal and corrects its pose to reduce the uncertainty.

The decision on how many iterations to perform depends on the number of matching lines which is impossible to know in advance. We terminate the loop after a constant number of iterations. Our justification for the number of iterations is given in the Appendix.

\section{EXPERIMENTS}

To demonstrate the functionality of the mobile robot, we performed a series of tests with the robot in an actual outdoor environment. Three kinds of tests were performed-one that aimed to evaluate the performance of the open-space localization method, another that focused only on the vision component, and a final test that used both methods. The open-space localization experiments were run on a relatively flat area and estimated the robot position and orientation in 2-D. The rest of the experiments were performed in 3-D estimating the full 6 DOF of the robot pose because the entire test area was not entirely flat.

\section{A. Localization in Open Space}

The purpose of these tests were to investigate the accuracy of the open space localization method described in Section III.

Arbitrary trajectories were generated and executed. The trajectories were piecewise linear, with the robot turning to its next target in place as soon as it reached the current one. The maximum translational and rotational velocities were $0.5 \mathrm{~m} / \mathrm{s}$ and $0.4 \mathrm{rad} / \mathrm{s}$, respectively.

To test the accuracy of the system, two comprehensive test runs were set up to obtain ground truth data. A piece of chalk was attached at the center of odometry on the bottom of the vehicle so that when the robot moved it plotted its actual trajectory on the ground. After it completed the task, sample points from the actual trajectory were marked at intervals of approximately $1 \mathrm{~m}$, and measurements of each sample point were obtained.

First, a complex desired trajectory of 14 targets and total length of $210 \mathrm{~m}$ was used. Fig. 9 shows the planned and actual trajectories, overlaid on the map of the test area. The average deviation of the robot from the planned trajectory over all sampled points in this run was $0.46 \mathrm{~m}$ with a maximum value of $0.94 \mathrm{~m}$.

The second trajectory consisted of nine targets arranged in the shape of the digit eight around the two planters in the center of Fig. 9. The trajectory was $132 \mathrm{~m}$ long and asked the robot to return to the same place where it started (Fig. 10). The average error for this run was $0.251 \mathrm{~m}$.

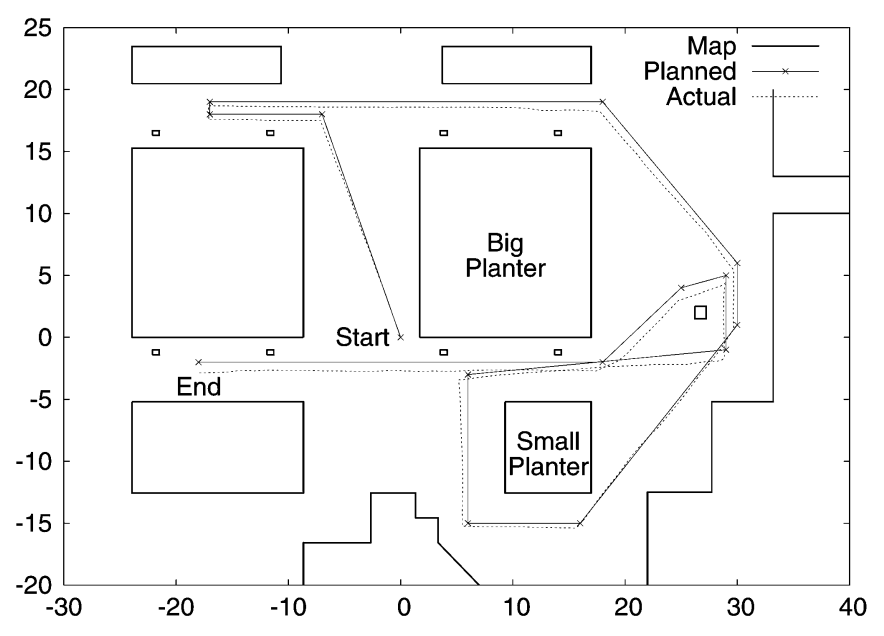

Fig. 9. First test run in open space.

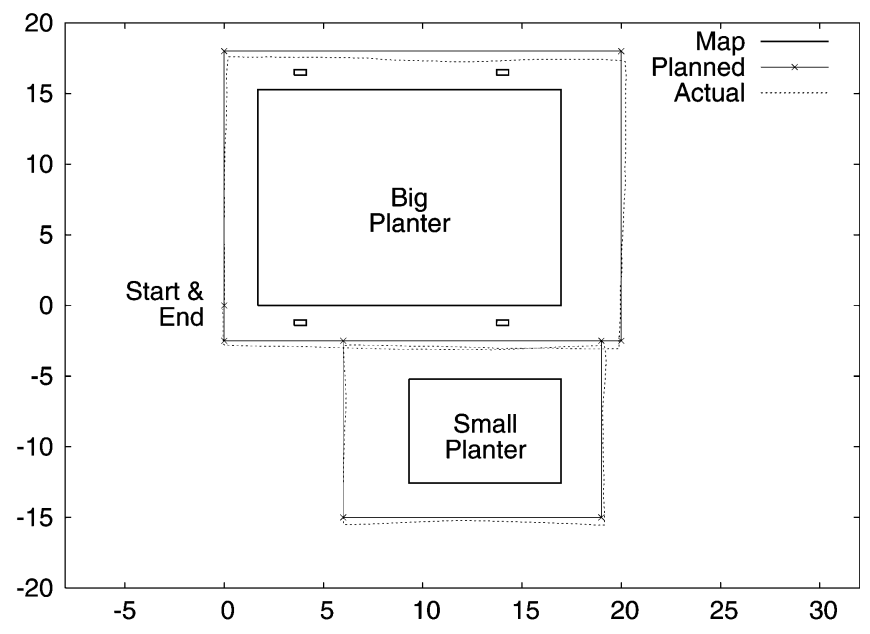

Fig. 10. Second open-space test run: returning to the starting point.

The next experiment also involved the trajectory in Fig. 10, but this time of interest was the displacement between the starting and arrival locations. Ideally, the robot had to arrive at its starting location since this was a closed-loop trajectory. Three such runs were performed. The resulting errors were $0.08,0.334$, and $0.279 \mathrm{~m}$.

It should be noted that the performance of the open-space localization system strongly depends on the accuracy of the GPS data. During the experiment above, the number of satellites used were six or seven most of the time, occasionally dropping to five or increasing to eight. The GPS receiver was working in RTK float mode in which its accuracy is worse compared to when it works in RTK fixed mode. The latter mode provides accuracy to within a few centimeters, however, it is typically available when seven or more satellites provide good signal-to-noise ratio (SNR) over a long period of time.

These results demonstrate that this localization method is sufficient for navigation in open areas with typical GPS performance and no additional sensors are needed in such cases. The location estimate errors in all of the above test runs were within the acceptable range for our urban site-modeling task. 


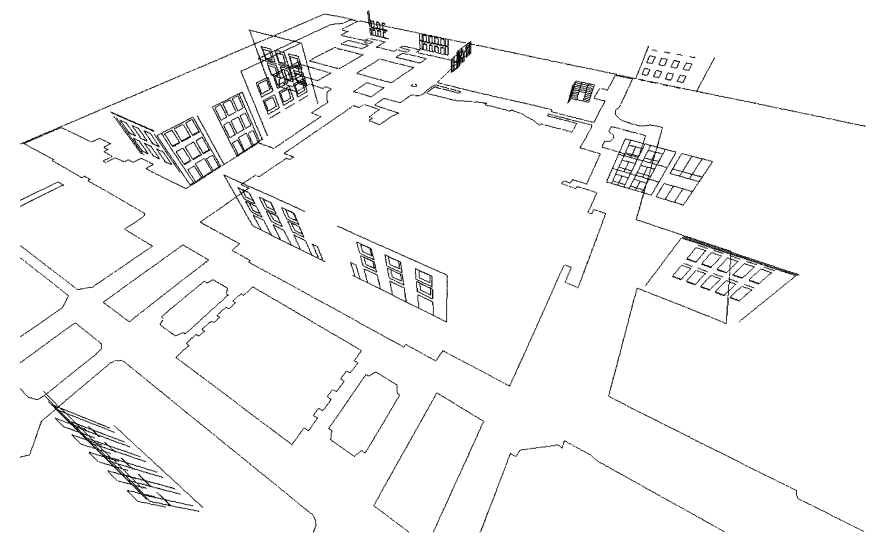

Fig. 11. Three-dimensional models used for localization shown on a 2-D map of the test area.

\section{B. Localization With Vision}

To examine the accuracy of the visual localization method, we performed two kinds of tests: one that compares the result for each test location with ground truth data and one that compares the two results the algorithm produced on two different images taken from the same location.

In both kinds of tests, we wanted to measure the quality of the location estimation alone and minimize the interference from inaccuracies in the model. Thus, we took care to create accurate models of the buildings used by scanning their prominent features with a high-quality electronic theodolite with a nominal accuracy of $2 \mathrm{~mm}$. The features modeled were windows, ledges, and decorations-all commonly found and abundant in urban structures and easy to find using 2-D image operators (Fig. 11). The model database consisted of 16 facades with the number of line segments ranging from 15 to 51 , averaging at 24 .

In the first test, the robot was driven along a long trajectory around a large building. At 16 relatively regularly spaced locations, the robot was instructed to stop and perform the visual localization routine. The robot used the accumulated error from odometry as an initial guess to determine the nearby buildings and choose a model of one for localization. A sketch of the test area with the test locations and directions in which the images were taken is shown in Fig. 12.

Fig. 13 shows the results of the 16 runs. The left image in each pair shows the model used projected onto the image using the initial inaccurate estimate of the camera pose. The image to the right shows the model projected on the image after the correct camera pose was computed. The number of matched features ranged between 12 and 25, with an average of 18 . In all cases, the alignment of the model and the image is very accurate.

Since it is extremely difficult to determine the location of the robot with a near centimeter-level accuracy, ground truth for the visual localization experiments at each location was obtained in the following manner: an electronic theodolite was placed near the robot and the building facade it was looking at. While the robot was stationary, a scan of the camera lens was taken with the theodolite. Then, a few key points of the building facade were also surveyed so that the location of the camera lens could be determined with respect to the building. Finally, the expected

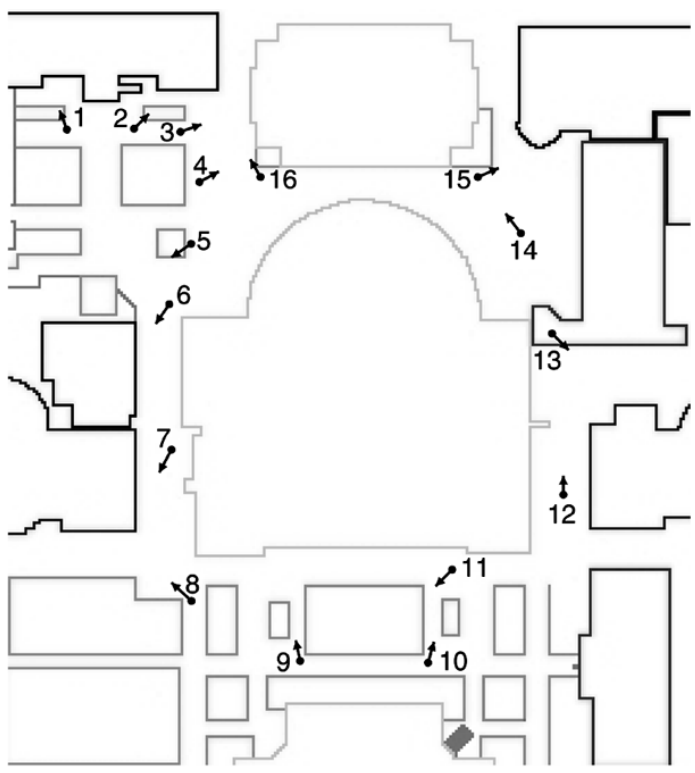

Fig. 12. Map of the area where the experiments were conducted, showing approximate camera locations and orientations.

location of the camera with respect to the building was computed based on the robot estimate of its pose, and it was compared with the one obtained by the theodolite.

Because of the size of the camera lens, the error introduced by scanning its surface, rather than the focal center, was less than $2 \mathrm{~cm}$, which is small in comparison with the errors of the algorithm. The resulting errors in translation were 0.081 $\mathrm{m}$ ( $\min ), 0.442(\max )$, and 0.268 (average). These errors are small and clearly demonstrate that the method generates accurate estimates that can be used for robot navigation in urban environments.

The alignment of model and image in the resulting poses suggests that the orientation is also estimated accurately. While this can be seen from Fig. 13, we wanted to obtain a quantitative confirmation. We did this by running Tsai's method for external camera parameters estimation [35] and comparing its orientation estimates with the ones from our localization algorithm. The resulting errors were within a fraction of a degree: $0.131^{\circ}$ (min), $0.977^{\circ}(\max )$, and $0.570^{\circ}$ (average).

The purpose of the second test was to confirm that the algorithm generates consistent results when used on different facades from the same location. We took two pairs of images from the same spot—one pair at location 4 and one pair at location 5-by simply panning and tilting the camera. Both pairs of images were processed with their corresponding models (Figs. 14 and 15) and were intentionally given initial pose estimates with large errors. The two results for each pair were compared with each other and revealed only small discrepancies: $0.064 \mathrm{~m}$ (location 4) and $0.290 \mathrm{~m}$ (location 5).

\section{Localization Using All Sensors}

Finally, a test was performed to confirm that the entire localization system works well together, that is, it uses the visual localization as needed and that it actually improves the performance. A more than 330-m-long trajectory was composed 

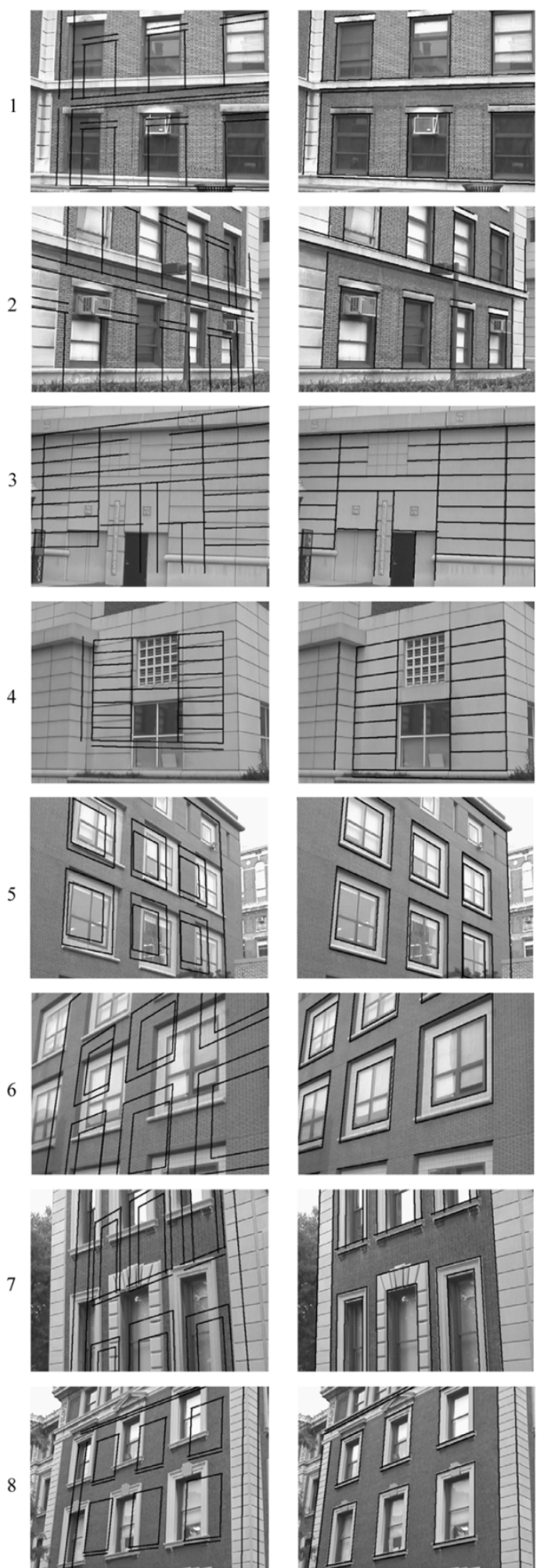
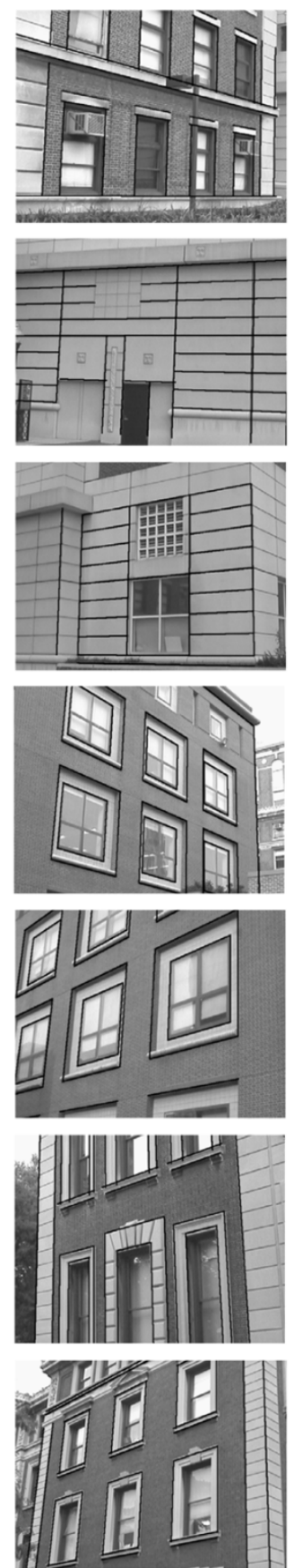
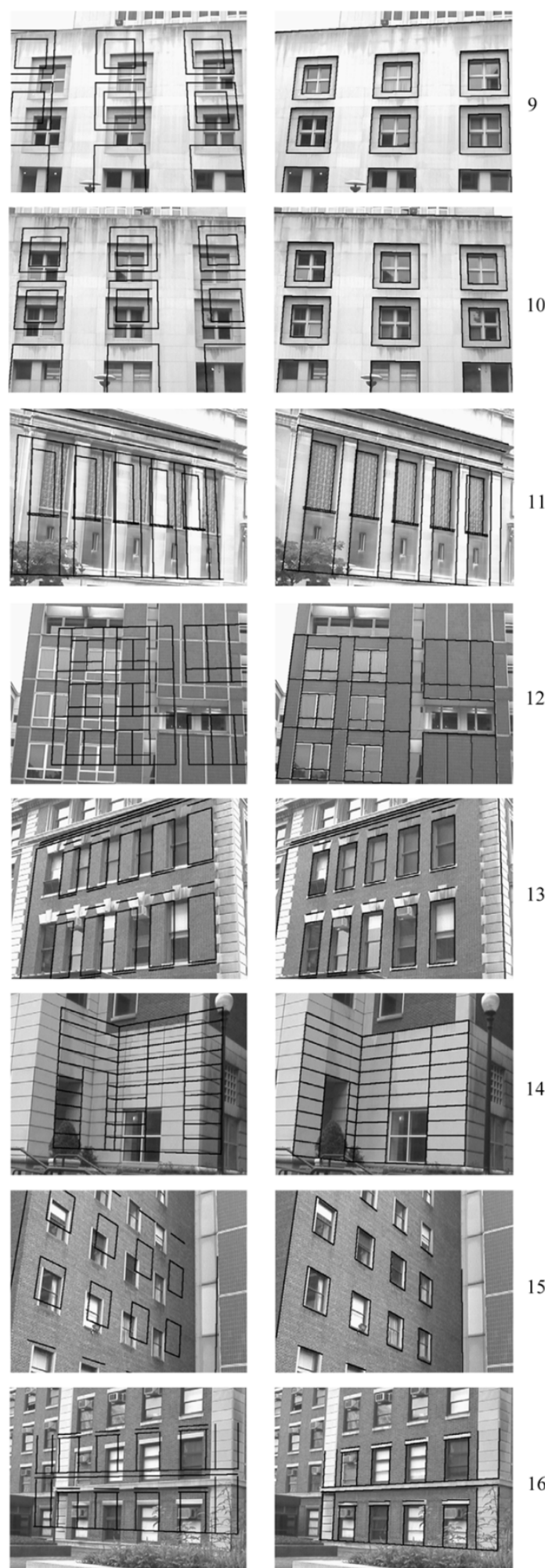


shows the rough estimate of the pose that was used to initiate the visual localization. The right image shows the resulting pose of the algorithm.

(Fig. 16), and the robot was directed to follow that trajectory using all sensors, including vision, as needed.

During the test run, the robot passed through both areas of good GPS visibility and poor GPS visibility. It was set up to seek visual localization whenever the standard deviation of the uncertainty of the current position exceeded $1 \mathrm{~m}$. The robot was consistently able to detect the areas of poor GPS performance (marked on Fig. 16) and supplement it with vision. Notice that no GPS data were available at all at location 3 , as the robot was directly beneath an extension of the nearby building.

The robot stopped at each marked location, correctly determined a nearby building to use, and performed the visual 

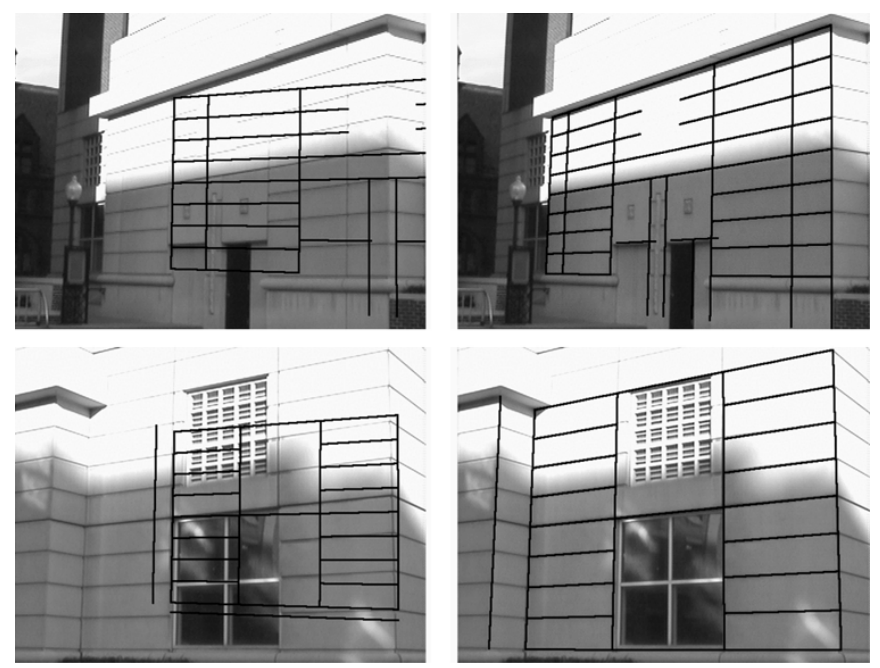

Fig. 14. Consistency test 1: Initial and final alignments in the pose estimation tests with a pair of images taken from the same location.
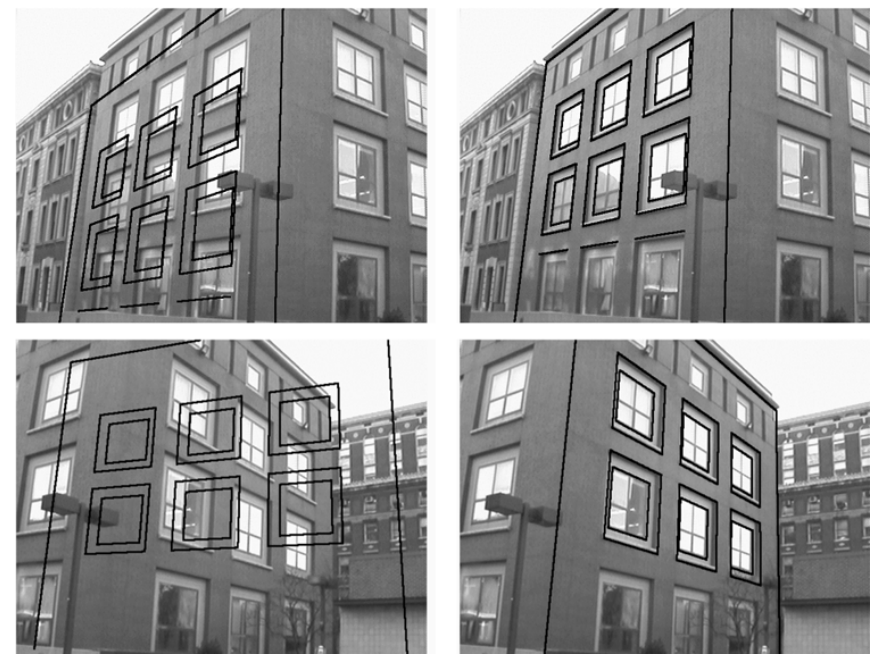

Fig. 15. Consistency test 2: Initial and final alignments in the pose estimation tests with a pair of images taken from the same location.

localization procedure described in Section IV. While at rest, we scanned its camera with an electronic theodolite to obtain ground truth.

Table I compares the estimates of the robot position at each location. The top line of each table row shows the estimate of the open-space localization method prior to triggering the visual procedure and its error. The bottom line of the same row shows the estimate and the error of the visual localization. The table clearly demonstrates the improvement the visual algorithm makes to the overall system performance. The corresponding images overlaid with the model are shown in Fig. 17.

\section{DISCUSSION AND FUTURE WORK}

This paper presented a practical approach to mobile robot localization in urban environments. The work was done as part of our AVENUE project for urban site modeling, however, the methods and ideas presented here are independent of the project and are generally applicable to mobile robots operating in urban environments.

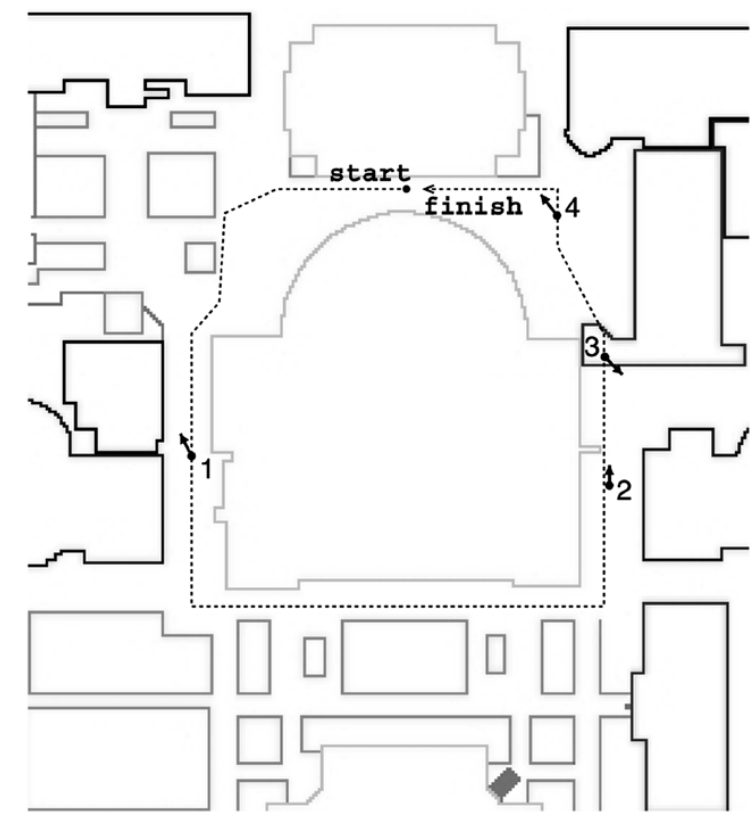

Fig. 16. Map of the area showing the robot trajectory (dotted line) and the locations where the robot used visual localization. Notice location 3, which is directly underneath a building extension.

TABLE I

ROBOT POSITION AND ERROR ESTIMATED BY GPS, COMPASS, AND ODOMETRY ALONG WITH THE CORRESPONDING IMPROVED POSITION ESTIMATE AND ERROR After Performing Visual Localization. Measurements are in Meters

\begin{tabular}{c|c|r|r|r||c}
\hline No & Type & \multicolumn{1}{|c|}{$X$} & \multicolumn{1}{|c|}{$Y$} & \multicolumn{1}{|c||}{$Z$} & Error \\
\hline \hline 1 & Open-space & 17.547 & -58.079 & -0.683 & 1.297 \\
& Vision & 16.470 & -58.565 & 0.110 & 0.348 \\
\hline 2 & Open-space & 108.521 & -61.634 & 0.279 & 1.031 \\
& Vision & 108.260 & -62.001 & 1.127 & 0.345 \\
\hline 3 & Open-space & 90.660 & -30.729 & 1.418 & 0.937 \\
& Vision & 91.344 & -31.404 & 0.867 & 0.179 \\
\hline 4 & Open-space & 95.095 & 2.577 & 1.713 & 1.212 \\
& Vision & 95.363 & 2.881 & 0.850 & 0.274 \\
\hline
\end{tabular}

The localization system employs the robot odometry, a digital compass with an integrated tilt sensor, a global positioning unit, and a camera mounted on a pan-tilt head in two complementary ways. The open-space localization method uses odometry, the digital compass, and GPS. The sensor integration is done by an extended Kalman filter. The method can be performed in real time. The visual localization method is heavier computationally but is only used upon demand. The pose estimation is done by matching an image of a nearby building with a simple and compact model. A database of the models is stored on the on-board computer. No environmental modifications are required. We have demonstrated the functionality of the robot and the localization methods with numerous experiments.

Our visual localization method raises an interesting question about the amount of time it takes on a given mission. This time is determined by the number of localization efforts as well as the time spent on each of them (currently, 25-45 s). The number of visual localization runs depends on a number of factors, such as the quality of the GPS fixes, the number of satellites seen, the number, shape, and look of the nearby buildings, and the accuracy and completeness of the model. Except for the model, these are factors which are beyond our control and therefore the 

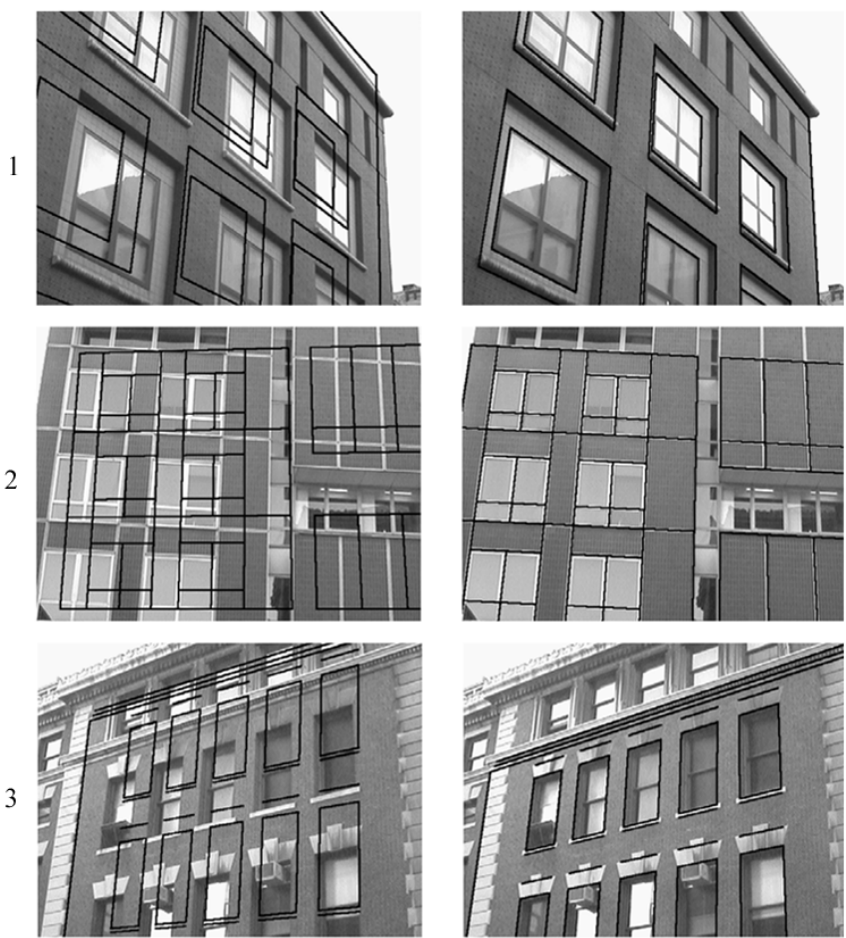

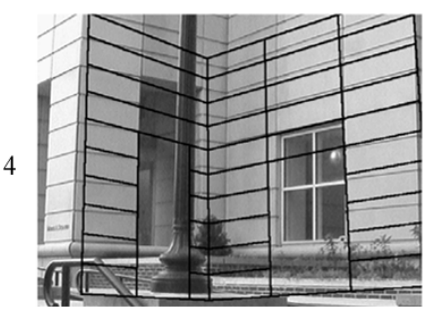

(a)

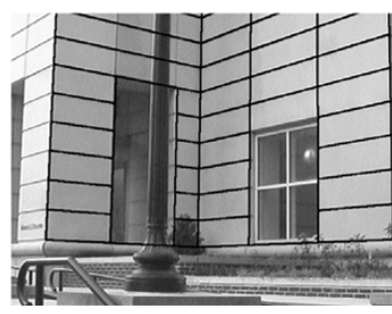

(b)
Fig. 17. Integration tests. (a) Initial estimates from open-space method and (b) resulting estimate of the visual localization method for the four locations in Fig. 16.

number of visual localization steps is expected to vary greatly depending on the particularities of the mission.

The time spent on a visual localization step is mainly determined by the number of features detected in the image and the number of features in the model. At least three matches are needed for the visual localization to produce a result. Increasing the number of features usually leads to better accuracy and stability up to a certain point at the price of more time spent on computation. A further increase of the number of features does not result in any appreciable improvement but adds to the computational burden. We have presented an analysis of the running time based on the number of features in the Appendix. However, we do not think there is a simple answer to the question of what the optimum number of features is because not all features are of equal importance. For example, the removal of some of the shorter lines on the model shown on Fig. 14 does not cause serious problems, while removing any of the longer lines causes the pose estimation to fail. Some facades contain a high degree of repetitiveness which may lead to a confusion in the matching process (Fig. 18). This suggests a heuristic which prefers longer over shorter lines and includes some unique (i.e., nonrepetitive) features. The existence of such features is not always guaranteed, however, and this is one limitation of our method.

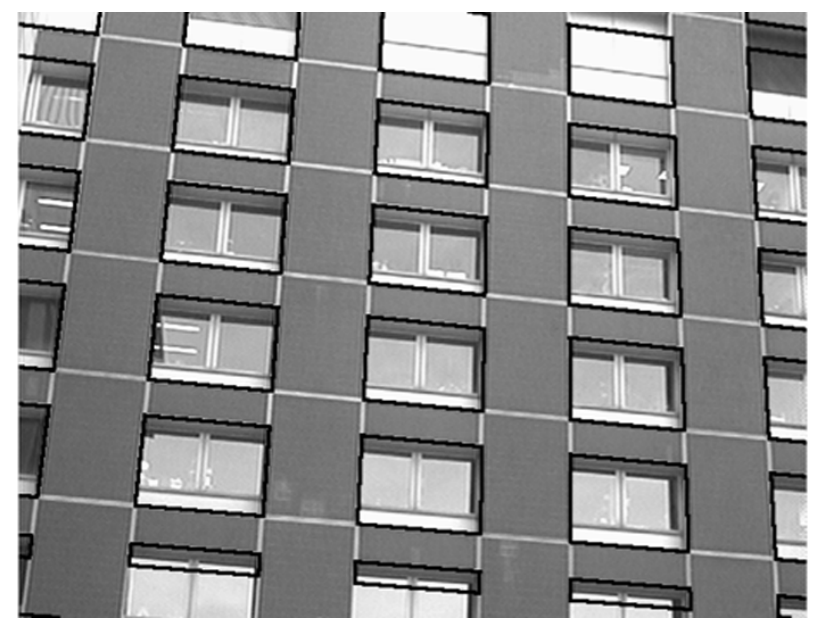

Fig. 18. Example of an incorrect match between image and model. The match would have been correct if the model were not shifted one flight up.

Another limitation of our current implementation is that it uses only one of the visible building facades even if more may be present. It is possible to extend the method to use all visible facades, however, this may not scale well given the considerations above of the running time, unless an additional constraint is imposed to speed up the matching process.

One last thing that we need to discuss is the way we obtain the environmental model used for the visual localization method. It is tightly coupled to the intended use of the method. Recall that the work presented here is part of a project whose goal is the creation of a detailed geometric and photometric 3-D model of an urban site. We refer to this detailed model as the detailed model, as opposed to the localization model used for localization.

The detailed 3-D models obtained from the range scans and images of the buildings are too large and complex since they capture a lot of detail [Fig. 19(b)]. The modeling process is incremental. At each stage, there is a partial model of the site available. When new range scans and images are acquired to be integrated with the existing partial detailed model, a data simplification step is done which creates a reduced complexity model for the purpose of the registration of the coordinate systems of the range scanner and the camera. This simplified model [Fig. 19(c)] consists of 3-D line segments obtained by segmenting the range scans into planar regions and intersecting planes to obtain lines (for details, see [36]). The result of this operation is a set of line segments- the kind that we need for visual localization.

Thus, to create a localization model, only some postprocessing is needed of the available 3-D line features. The set of lines need to be broken into near-planar regions and a representative coordinate system needs to be established for each such region. This is the focus of our current efforts to complete the integration between the modeling and the localization aspects of our project.

Note that there is no controversy here about which model comes first (the bootstrapping problem). The robot will start from a certain location, scan the buildings in view, create their partial detailed models, and register them with its original pose. As a result, localization models of some of the scanned facades will become available which the robot may use for navigation 


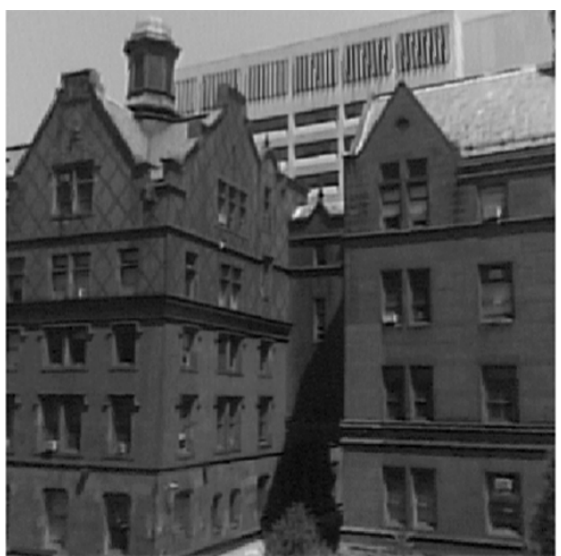

(a)

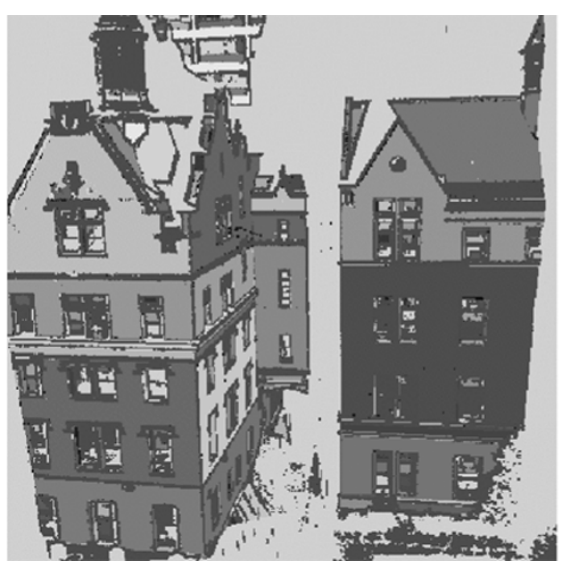

(b)

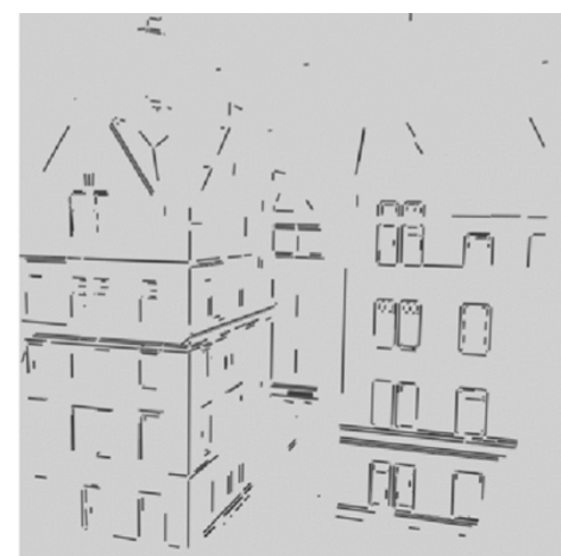

(c)

Fig. 19. Model acquisition and simplification. (a) An image of a building. (b) The 3-D model created from the image and a range scan. (c) A reduced version of the same model consisting only of line features.

to its next scanning destination. As more new scans and images are obtained and the detailed model gets updated, more localization models will become for use by the robot on its way farther along its modeling path.

\section{APPENDIX \\ NUMBER OF ITERATIONS AND SPEEDUPS IN THE VISUAL METHOD}

The decision on how many iterations to perform is based on the expected number of trials $k_{r}$ required to obtain a correct match. If the number of line segments obtained from the image is $n_{2}$, the number of line segments in the model is $n_{3}$, and $n$ of them appear in both the model and the image, then the probability of a single sample being correct is

$$
p=\frac{n}{n_{3}} \frac{(n-1)}{\left(n_{3}-1\right)} \frac{(n-2)}{\left(n_{3}-2\right)} \frac{1}{n_{2}} \frac{1}{\left(n_{2}-1\right)} \frac{1}{\left(n_{2}-2\right)} .
$$

The expected value $E\left(k_{r}\right)$ of the number of trials is then

$$
E(k)=\sum_{i=1}^{\infty} i p(1-p)^{i-1}=\frac{1}{p} .
$$

We see that $E(k)$ depends on the number of matching line segments which is impossible to know in advance. Our approach is to use a fixed number of iterations which is determined on the basis of the number of lines in the model and the average number of edge lines used in the pose computation step. This number can be controlled to a large degree by choosing an appropriate threshold in the reduction steps described below.

Typically, $E(k)$ is a computationally prohibitive number and we take a number of steps to make it tractable. The first step is to merge all collinear line segments in both the 3-D line set and the 2-D line set. This ensures a one-to-one match between the two sets and eliminates a great number of practically equivalent combinations.

Next, we notice that short lines are not as informative as long ones, as a slight perturbation of the endpoints of a short line (for example, due to misdetected edgels) could lead to large change in its orientation. Therefore, we discard line segments that are shorter than a threshold, thus further reducing the value of $E(k)$.
Additional decrease of the number of expected iterations is achieved by splitting the line segments into two disjoint groups: mostly horizontal and mostly vertical ones. This is easy to do for the lines from the model, since the information is directly available. It is also possible to do it for the edge lines, because the tilt of the robot is accurately measured by the digital compass module and the building facade is assumed to be a vertical, near planar surface. Misclassifications of edge lines are possible but extremely rare and normally do not affect the accuracy of the algorithm.

The benefit of splitting the segments into two groups is to eliminate samples that are certain to be incorrect matches such as ones that associate a horizontal line on the model with a vertical one in the image. The sampling step is modified to always produce samples having one pair of mostly horizontal lines and one pair of mostly vertical lines. The third pair could be from either class.

For typical values, such as $n_{2}=30, n_{3}=24$, and $n=18$ after the elimination steps described above, with an approximately equal number of horizontal lines and vertical lines, the expected number of iterations becomes less than 7553 . This already is a practical number. For comparison, all visual localization tests in this paper used a maximum of 8000 iterations, which typically took between 25 and $45 \mathrm{~s}$ on a $2-\mathrm{GHz}$ Pentium IV processor equipped with $1 \mathrm{~GB}$ of RAM.

\section{REFERENCES}

[1] P. Allen, I. Stamos, A. Gueorguiev, E. Gold, and P. Blaer, “AVENUE: Automated site modeling in urban environments," in Proc. 3rd Conf. Digital Imaging and Modeling, Quebec, QC, Canada, May 2001, pp. 357-364.

[2] J. Borenstein and L. Feng, "Correction of systematic odometry errors in mobile robots," in Proc. IROS'95, Pittsburgh, PA, Aug. 1995, pp. $569-574$.

[3] - "Gyrodometry: A new method for combining data from gyros and odometry in mobile robots," in Proc. IEEE Int. Conf. Robotics and Automation, Minneapolis, MN, 1996, pp. 423-428.

[4] S. I. Roumeliotis, G. S. Sukhatme, and G. A. Bakey, "Circumventing dynamic modeling: Evaluation of the error-state Kalman filter applied to mobile robot localization," in Proc. IEEE Int. Conf. Robotics and Automation, Detroit, MI, 1999, pp. 1656-1663.

[5] S. Cooper and H. F. Durrant-Whyte, "A Kalman filter model for GPS navigation of land vehicles," in Proc. IEEE Int. Conf. Robotics and Automation, San Diego, CA, 1994, pp. 157-163. 
[6] J. Schleppe, "Development of a real-time attitude system using a quaternion parametrization and non-dedicated GPS receivers," Master's thesis, Dept. Geomatics Eng., Univ. Calgary, Calgary, AB, Canada, 1996.

[7] T. Aono, K. Fujii, S. Hatsumoto, and T. Kamiya, "Positioning of vehicle on undulating ground using GPS and dead reckoning," in Proc. IEEE Int. Conf. Robotics and Automation, Leuven, Belgium, 1998, pp. 3443-3448.

[8] C.-C. Lin and R. L. Tummala, "Mobile robot navigation using artificial landmarks," J. Robot. Syst., vol. 14, no. 2, pp. 93-106, 1997.

[9] S. Thrun, "Finding landmarks for mobile robot navigation," in Proc. IEEE Int. Conf. Robotics and Automation, Leuven, Belgium, 1998, pp. 958-963.

[10] R. Sim and G. Dudek, "Mobile robot localization from learned landmarks," in Proc. IEEE/RSJ Int. Conf. Intelligent Robots and Systems, Victoria, BC, Canada, Oct. 1998, pp. 1060-1065.

[11] A. Kosaka and A. C. Kak, "Fast vision-guided mobile robot navigation using model-based reasoning and prediction of uncertainties," Comput. Vis., Graphics, Image Process., Image Understand., vol. 56, no. 3, pp. 271-329, 1992.

[12] M. Bozorg, E. Nebot, and H. Durrant-Whyte, "A decentralised navigation architecture," in Proc. IEEE Int. Conf. Robotics and Automation, Leuven, Belgium, 1998, pp. 3413-3418.

[13] J. Neira, J. Tardos, J. Horn, and G. Schmidt, "Fusing range and intensity images for mobile robot localization," IEEE Trans. Robot. Automat., vol. 15, pp. 76-84, Feb. 1999.

[14] J. Castellanos and J. Tardos, Mobile Robot Localization and Map Building: A Multisensor Fusion Approach. Norwell, MA: Kluwer, 1999.

[15] S. Thrun, W. Burgard, and D. Fox, "A probabilistic approach to concurrent mapping and localization for mobile robots," Auton. Robots, vol. 5, pp. 253-271, 1998 .

[16] F. Dellaert, D. Fox, W. Burgard, and S. Thrun, "Monte Carlo localization for mobile robots," in Proc. IEEE Int. Conf. Robotics and Automation, Detroit, MI, 1999, pp. 1322-1328.

[17] H. Durrant-Whyte, G. Dissanayake, and P. Gibbens, "Toward deployment of large scale simultaneous localization and map building (SLAM) systems," in Proc. Int. Symp. Robotics Research, Salt Lake City, UT, 1999, pp. 121-127.

[18] J. Leonard and H. J. S. Feder, "A computationally efficient method for large-scale concurrent mapping and localization," in Proc. Int. Symp. Robotics Research, Salt Lake City, UT, 1999, pp. 128-135.

[19] J. Borenstein and L. Feng, Navigating Mobile Robots: Systems and Techniques. Wellesley, MA: A. K. Peters, 1996.

[20] H. R. Everett, Sensors for Mobile Robots: Theory and Application. Wellesley, MA: A. K. Peters, 1995.

[21] D. Kortenkamp, R. P. Bonasso, and R. Murphy, Artificial Intelligence and Mobile Robots: Case Studies of Successful Robot Systems. Cambridge, MA: AAAI Press/MIT Press, 1998.

[22] G. Dissanayake, P. Newman, S. Clark, H. Durrant-Whyte, and M. Csorba, "A solution to the simultaneous localization and map building (SLAM) problem," IEEE Trans. Robot. Automat., vol. 17, pp. 229-241, June 2001.

[23] J. Guivant, E. Nebot, and H. Durrant-Whyte, "Simultaneous localization and map bidding using natural features in outdoor environments," Intell. Auton. Syst. 6, vol. 1, pp. 581-588, July 2000.

[24] R. Talluri and J. K. Aggarwal, "Mobile robot self-location using modelimage feature correspondence," IEEE Trans. Robot. Automat., vol. 12, pp. 63-77, Feb. 1996.

[25] T. Chen and R. Shibasaki, "High precision navigation for 3-D mobile GIS in urban area by integrating GPS, gyro and image sequence analysis," in Proc. Int. Workshop Urban 3D/Multi-Media Mapping, Tokyo, Japan, 1999, pp. 147-156.
[26] R. Nayak, "Reliable and continuous urban navigation using multiple GPS antennas and a low cost IMU," master's thesis, Dept. Geomatics Eng., Univ. of Calgary, Calgary, AB, Canada, 2000.

[27] I. Stamos, "Geometry and texture recovery of scenes of large scale," $J$ Comput. Vis. Image Understand., vol. 88, no. 2, pp. 94-118, Nov. 2002.

[28] P. Blaer, "Robot Path Planning Using Generalized Voronoi Diagrams,", http://www.cs.columbia.edu/ pblaer/projects/path-planner.

[29] E. Gold, "AvenueUI: A comprehensive visualization/teleoperation application and development framework for multiple mobile robots," Master's thesis, Dept. Comput. Sci., Columbia Univ., New York, NY, 2001.

[30] R. Brown and P. Hwang, Introduction to Random Signals and Applied Kalman Filtering, 3rd ed. New York: Wiley, 1997.

[31] A. Georgiev, "Design, implementation and localization of a mobile robot for urban site modeling," Ph.D. dissertation, Columbia Univ., New York, NY, 2003.

[32] M. Fischler and R. Bolles, "Random sample consensus: A paradigm for model fitting with applications to image analysis and automated cartography," in DARPA, 1980, pp. 71-88.

[33] R. Kumar and A. R. Hanson, "Robust methods for estimating pose and a sensitivity analysis," Comput. Vis. Graph. Image Process., vol. 60, no. 3, pp. 313-342, Nov. 1994.

[34] B. K. P. Horn, "Relative orientation," Int. J. Comput. Vis., vol. 4, no. 1, pp. $59-78,1990$.

[35] R. Y. Tsai, "A versatile camera calibration technique for high-accuracy 3-D machine vision metrology using off-the-shelf TV cameras and lenses," J. Robot. Automat., vol. RA-3, pp. 323-344, Aug. 1987.

[36] I. Stamos and P. K. Allen, "Integration of range and image sensing for photorealistic 3D modeling," in Proc. IEEE Int. Conf. Robotics and Automation, San Francisco, CA, 2000, pp. II:1435-1440.

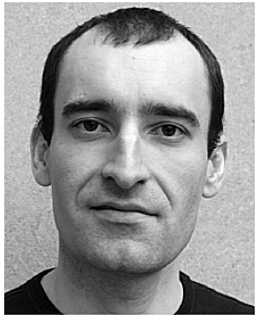

Atanas Georgiev (M'04) received the the M.S. degree in computer science from Sofia University "St. Kliment Ohridski," Sofia, Bulgaria, and the M.S., M.Phil., and Ph.D. degrees in computer science from Columbia University, New York, NY.

$\mathrm{He}$ is a Postdoctoral Research Scientist with Columbia University. His current research interests are in the areas of mobile robots and robotic micromanipulation.

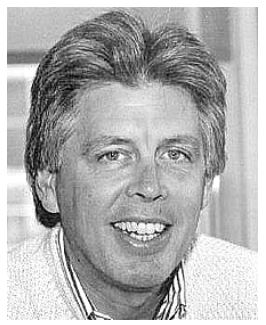

Peter K. Allen (S'82-M' 85) received the A.B. degree from Brown University, Providence, RI, in mathematics/economics, the M.S. degree in computer science from the University of Oregon, Eugene, and the $\mathrm{Ph} . \mathrm{D}$. degree in computer science from the University of Pennsylvania, Philadelphia.

$\mathrm{He}$ is a Professor of Computer Science with Columbia University, New York, NY. His current research interests include real-time computer vision, dextrous robotic hands, three-dimensional modeling, and sensor planning.

Prof. Allen has been named a Presidential Young Investigator by the National Science Foundation 